\title{
Improvement of a car racing controller by means of Ant Colony Optimization algorithms
}

\author{
Luis delaOssa, José A. Gámez and Verónica López
}

\begin{abstract}
The performance of a car racing controller depends on many factors. Although there are strong dependencies among these, some subproblems intrinsic to the design process can be addressed independently. Thus, if the aim is to minimize the lap time on a track, it becomes necessary to find the right trace around it and to determine the maximum speed on each stretch.

This study proposes a hybrid solution to achieve this. Traces, which are described as a configuration of points the car must move towards, are found by means of an Ant Colony Optimization algorithm, the Ant System. As the maximum speed strongly depends on such traces, it must be adjusted for each one of them. In order to do this, a local search procedure is used in two ways: either when the best trace has been found, or during the search for such a trace.

Results show a significant improvement with this technique in comparison with the original heuristic controller in terms of lap time. Moreover, the number of laps required for the algorithms to reach the solution makes them viable as a learning mechanism in real-time simulation environments.
\end{abstract}

\section{INTRODUCTION}

Car Racing competitions are becoming increasingly popular in the field of computational intelligence [1], [2]. Basically, participants design controllers for the simulated cars by using some of the existing techniques, and compete in races or other contests. Part of the success of these events is due to the fact that they constitute a mid point between theory and real world applications.

There are some recent studies which use evolutionary computation to build car controllers. Thus, evolution strategies are used in [3] to set the weights of the Multilayer Perceptron which guides the car. This work is extended in [4] so that some parameters of the simulation environment are also evolved. Other approaches, such as Genetic Programming, have also been used [5].

In the above cases, the process is automatic to a certain extent, as a basic representation for the controllers (Neural Network or GP Tree) is defined, and then the controller is generated by means of evolution. Controller design, however, can be tackled from a different perspective: dividing the problem into several subproblems and addressing each one of them separately.

This work is based on this latter approach, and proposes a method for improving a base controller so that it reduces the lap time on a known circuit.

Luis delaOssa, José A. Gámez and Verónica López are with the Computing Systems Department at University of Castilla-La Mancha, Spain (phone: +34 967599200 (2413); fax: +34 967599224 ; email: ldelaossal jgamez@dsi.uclm.es).
There are many factors which have a direct influence on controller performance. However, once it implements the basic functionalities and is able to complete laps in an acceptable time without colliding, two of them become essential to reduce such time: the trace around the track and the maximum speed on each stretch.

The aim of this study is to provide the base controller with a mechanism which allows it to learn, after a few laps, both the right trace and the suitable speed.

Ant Colony Optimization (ACO) [6] algorithms have been widely used to solve problems which involve finding paths. Their main advantage in relation with other techniques is that they use heuristic information to make the search more efficient. In our proposal, one of these algorithms, the Ant System [7] has been used to determine the key points which describe a trace.

With regards to the speed maximization, this can be performed by means of a simple local search. However, as will be explained below, it strongly depends on the trace. Thus, it is necessary to optimize path and speed at the same time. In order to do this, two alternatives have been considered. In the first one, the speed is optimized once the best path has been found. In the second one, the speed is optimized for each path.

This paper is divided into six sections besides this introduction. First of all, Section II describes the simulation environment. Then, a first approach to the problem of time minimization is given in Section III. Section IV briefly introduces the Ant System, the ACO algorithm which has been used in this study, and Section V shows the way it has been used to find the right trace. Afterwards, Section VI explains the hybrid algorithm proposed to optimize the speed. Finally, Section VII offers some conclusions and the course for future work.

\section{THE CAR RACING SIMULATOR}

There are several car racing simulators which enable the creation of creating bots or controllers which can compete. One of them, TORCS [8], was used as the base platform for the contest which was held at WCCI 2008 [2], and it seems to be the new standard for car racing competitions.

However, in the last few years, the simulator proposed in [9] has been the most popular. This software, implemented in Java, simulates a radio RC Car in a 2D environment (Fig. 1). 


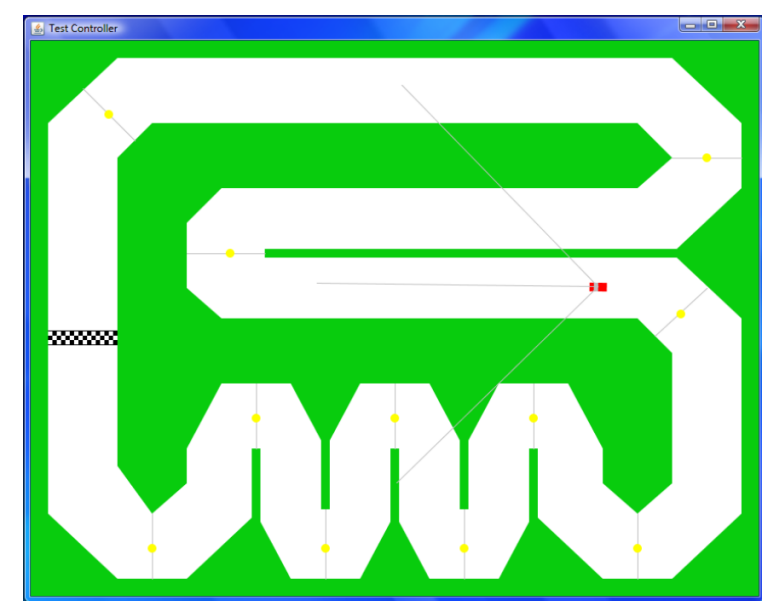

Fig. 1. Screenshot of the car racing simulator

This simulation environment consists of four basic parts:

- The car. It models the physical behaviour of the original car, whose movement is described by basic equations which consider its properties (mass, maximum turning radius, etc.), and two variables: the steer radius and power.

- The circuits. They are basically composed of an area the car can move across, and some shapes which delimit non-transitable zones.

Moreover, some guide points (called aimpoints) can be used in order to define a path. In our setting, aimpoints are located in the middle of each curve.

- The sensors. These allow detection of how far away the obstacles (limits of the circuit) are. Both their precision and position can be configured.

- The controllers. At each step of simulation, they read state information and produce the values for steer radius and power which are used by the car to calculate its new position. The input information is composed of:

- Distance to the obstacles detected by each one of the (three) sensors.

- Speed of the car.

- Angle to the next aimpoint.

- Angle of the curve, which is the angle between the line from the car to the next aimpoint, and the line from such a point to the following one (Fig. 2).

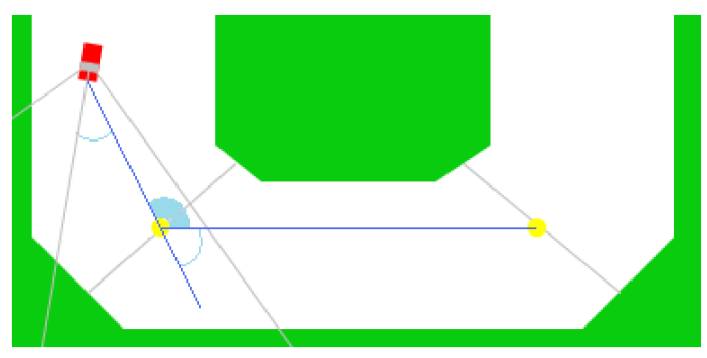

Fig. 2. Angle of the curve

\section{A FIRST APPROACH TO THE PROBLEM}

As mentioned above, one of the ways of dealing with controller design consists of problem decomposition. This means dividing the problem into subproblems, and tackling them in a semi-independent way. With this approach, a base controller is needed so that successive improvements can be tested.

In this study, the initial controller was designed heuristically so that it completes laps in a reasonable time without crashing into the walls of the road. In order to do this, it moves towards the next aimpoint trying to border the walls when the sensors detect them on the way. Then, when it reaches the point, it steers towards the next one.

There are many features of the base controller which should be studied in order to reduce lap times. However, we have focused on two of them that become essential once the base controller performs acceptably: path optimization and speed improvement.

It is obvious that speed must be increased as much as possible to minimize the lap time. Therefore, it becomes important to find the maximum speed on each stretch of the track. In particular, speed is limited in the curves because of the centrifugal force. Thus, the smaller the radius of the curve, the lower the speed the car can reach without coming off the track.

For simplicity, the base controller gives the maximum power on straights, but adjusts it so that the car reaches the right speed at the entry point of each curve. This speed is obtained as a function of the angle of the curve, and has been experimentally set at:

$$
\text { speed }=-2.35 \cdot \mid \text { CurveAngle } \mid+8.5
$$

Despite the fact that this estimation is very conservative and prevents the car from crashing into the walls, it allows the car to reach a relatively high speed. However, it could be noticeably improved.

On the other hand, the maximum speed at a certain curve not only depends on its angle, but also on the point where the car enters to it.

Curves can be entered at several points which go from the inside to the outside of the road. Although positioning the car on the outside part implies covering a bigger distance, it is worth doing so in most cases, since it allows higher speeds.

In this case, we have only considered the inside, the middle and the outside points to enter each curve, since previous experiments show that, for this environment, it is not worth using more.

Although there could be more alternatives, the set of the entry points to each curve can be used to describe a path around the track. The trajectory on the straight parts would also be determined by these points. Even the path at any given curve is determined, as can be seen in Figure 3, by both the entry point to the curve and the entry point to the next one. 


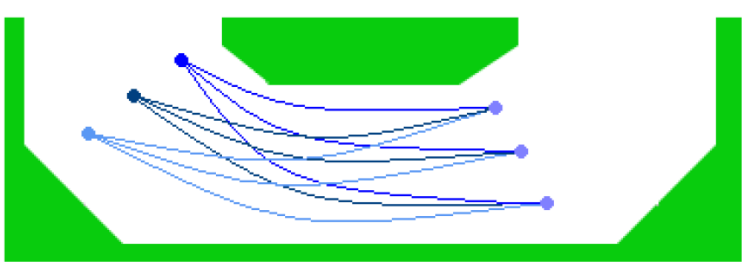

Fig. 3. Different trajectories between two curves

The set of paths for a circuit could be depicted, if considering the entry point to the curves, as the directed graph in Figure 4, where each one of the $N$ curves, $c$, is represented by a set of three nodes $L_{c}, C_{c}, R_{c}$ for the left, center, and right side respectively.

A path would then be described as a set $\left\{p_{1}, \ldots, p_{c}, \ldots, p_{N}\right\}$ of $N$ points, such that $p_{c} \in\left\{L_{c}, C_{c}, R_{c}\right\}$.

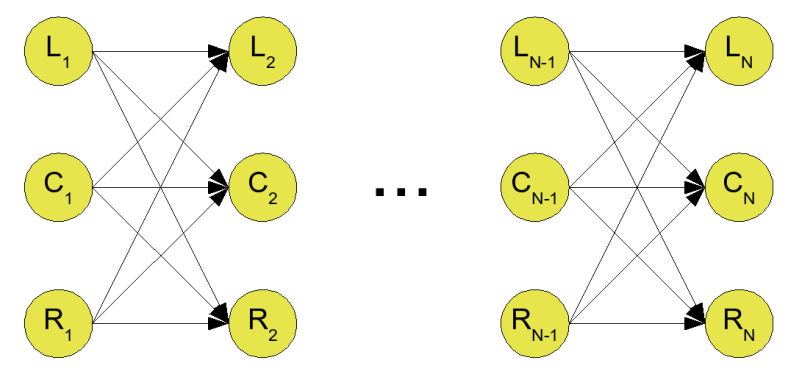

Fig. 4. Representation of the problem as a directed graph

Therefore, one of the problems for minimizing the lap time on a known track could be stated as finding a good path $P$, and determining the maximum speed at each one of the points $p_{c} \in P$. Since $p_{c}$ and the speed are dependent factors, both problems should be solved at the same time.

Lastly, it is worth pointing out that two modifications were done to the base controller in order to test the different configurations:

- First, instead of moving towards the aimpoint at each curve $c$, the car will move to the entry point $p_{c} \in P, P$ being the path which is being evaluated.

For simplicity, the location of the entry points $L_{1}, C_{1}, R_{1}, \ldots, L_{N}, C_{N}, R_{N}$, as well as that of the aimpoints, are provided as part of the circuit information. However, they could also be determined dynamically with the help of the sensors.

- Secondly, instead of using the expression in equation 1 , the speed at each point $p_{c} \in P$ will also be provided to the controller by the optimization algorithm.

\section{THE ANT SySTEM}

Ant Colony Optimization (ACO) algorithms [6] are techniques inspired by the behaviour of natural ants. As is known, these insects are able to find the shortest path between their nest and the place where they have found food [10]. The way they do this is called stigmergy. Basically, ants move randomly, but tend to go along the tracks where there is a larger amount of pheromones, which are chemical substances they deposit while they move. Since the concentration of pheromones increases on shorter paths, so does the proportion of ants that follow them.

ACO algorithms use a similar mechanism to tackle problems which involve finding paths on graphs.

Let $G$ be a directed graph composed of a set of nodes $N$ and a set of edges $E$, and let $i, j \in N$. In ACO, each edge $(i, j) \in E$ contains an initial amount of pheromone $\tau_{i j}(0)$, and is characterized by an heuristic value $\eta_{i j}$ which is fixed according to the problem. In the TSP problem, for instance, this value would be inversely proportional to the distance between cities represented by nodes $i$ and $j$ [6].

There is a huge number of ACO algorithms [6], [7], [11], [12]. All of them perform an iterative cycle which consists of two main procedures:

- Path building. The ants complete the paths according to the information contained on the edges.

- Pheromone updating. The amount of pheromones is updated according to the quality of the solutions (paths) found so far. This can be done once all ants have built a path, or as they go along, during the completion of the path.

In the algorithm used in this work, the Ant System (AS) [7], path completion and pheromone updating are carried out sequentially at each iteration $t$.

First of all, each one of the $m$ ants is placed at a random node, and then it moves along the edges until a path is completed (a valid solution to the problem is found). In order to build the path, ants consider both the heuristic information and the memoristic component, which is determined by the amount of pheromones.

Let $i$ be the current node, and $J_{i}^{k}$ all nodes ant $k$ can visit from node $i$. The next node in the path $j \in J_{i}^{k}$ is chosen with a probability:

$$
p_{i j}^{k}=\frac{\left[\tau_{i j}(t)\right]^{\alpha} \cdot\left[\eta_{i j}\right]^{\beta}}{\sum_{l \in J_{i}^{k}}\left[\tau_{i l}(t)\right]^{\alpha} \cdot\left[\eta_{i l}\right]^{\beta}}
$$

where $p_{i j}^{k}$ is the probability of ant $k$ moving from node $i$ to node $j ; \tau_{i j}(t)$ is the amount of pheromone on edge $(i, j)$ at iteration $t ; \alpha$ the importance of the memoristic component; $\eta_{i j}$ the heuristic preference of edge $(i, j)$; and $\beta$ the relative importance of the heuristic component.

Once all ants have built a path, the amount of pheromones on each edge is updated. This process is divided into two steps:

- In the first one, pheromone levels decay on all edges according to a factor $\rho$, as follows:

$$
\tau_{i j}(t+1)=(1-\rho) \cdot \tau_{i j}(t)
$$

- Afterwards, each edge receives an amount of pheromone $\Delta \tau_{i j}(t)=\sum_{k=1}^{m} \Delta \tau_{i j}^{k}(t)$, where $\Delta \tau_{i j}^{k}(t)$ is an amount of pheromone which depends on the quality of the solution found by ant $k$. 
Notice that, if the path built by ant $k$ does not include the edge $(i, j), \Delta \tau_{i j}^{k}(t)=0$.

A scheme for the Ant System process is shown in Fig. 5.

\section{Ant System}

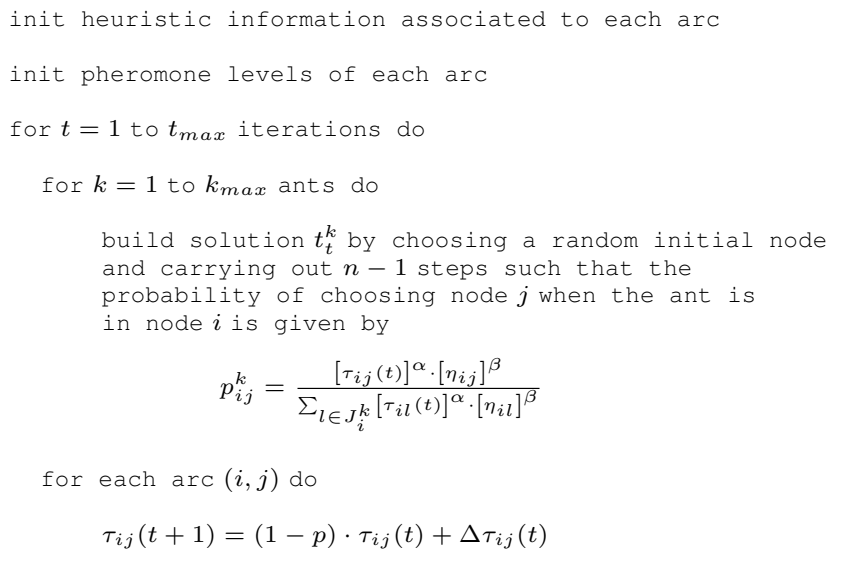

Fig. 5. Ant System Process

\section{TRACE OPTIMIZATION By MEANS OF ACO}

Let $P$ be a path of those contained in $G$. Its goodness is obtained by measuring the lap time when the car follows the trace it represents. In order to do this, the base speed at each one of the points is calculated according to Equation 1, as in the base controller. Moreover, a modification has been made so that the position at the curve can be utilized. If the entry point at curve $c, p_{c}$, is the inside one, the base speed is decreased by $30 \%$, whereas if $p_{c}$ is located at the outside part of the track, then the speed is increased by $30 \%$. These values have been set up heuristically after several tests and, as with the estimation of the base speed, the objective is to attain a high value, while preventing the car from coming off the track.

As mentioned above, ACO algorithms are suitable for solving problems which involve finding paths on graphs. Since they need a heuristic component, they are specially attractive when this information is available, as is the case in this problem.

In this particular, taking the curves on the outside is preferred, since it allows a higher speed which, generally, compensates for the bigger distance. After some preliminary tests, and in order to avoid premature convergence, a slight preference was set for such points. Thus, heuristic information has been set up as follows:

- $\eta_{p_{c} p_{c+1}}=0.4$, if $p_{c+1}$ represents the point on the outside part of the curve.

- $\eta_{p_{c} p_{c+1}}=0.3$, if $p_{c+1}$ represents the middle or the inside part of the curve.

The rest of parameters for the Ant System have been fixed as follows:

- Number of ants $=20$

- Importance of the heuristic information $\beta=1$.

- Memoristic information: $\tau_{i j}=0.5$ and $\alpha=0.15$.

- Evaporation $\rho=0.2$

- Maximum number of iterations $=150$.

As for the amount of pheromone deposited by each ant, this was fixed at:

$$
\frac{T_{\text {baseController }} * 1.01-T_{\text {ant }}}{500}
$$

where $T_{\text {baseController }}$ is the lap time obtained with the base controller and $T_{a n t}$ is the time recorded by the car when the controller follows the trace described by an ant.

In order to test this method, 8 different circuits with different levels of complexity were used (Figure 6). For each one of them, the Ant System algorithm was run 30 times.

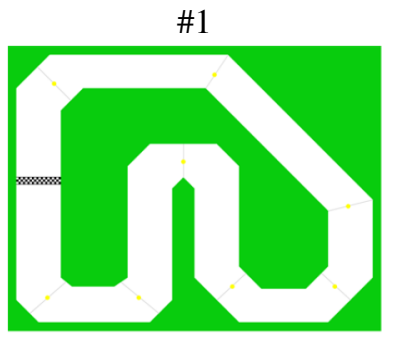

\#3

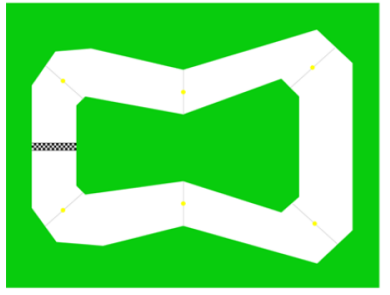

\#5

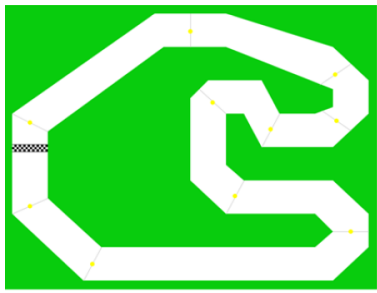

\#7

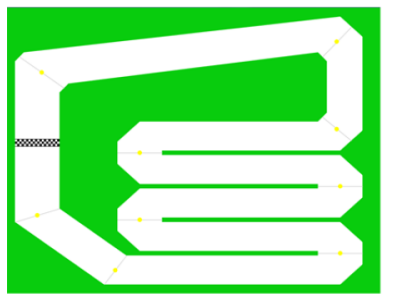

Fig. 6. Circuit used for the experimental evaluation

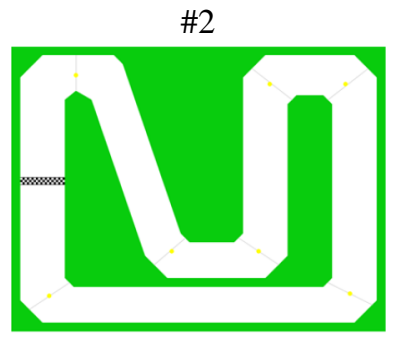

\#4

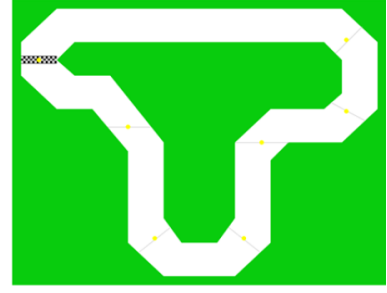

\#6

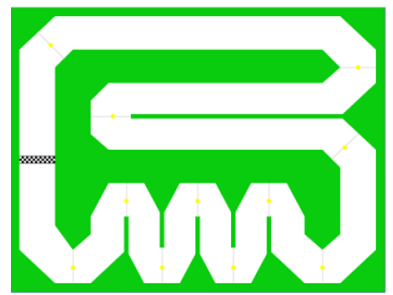

\#8

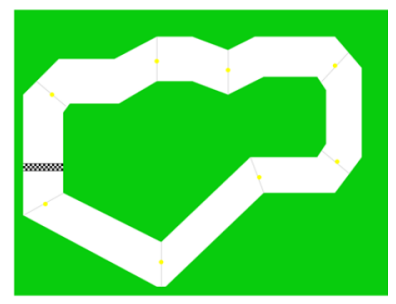


TABLE I

RESULTS OBTAINED FOR THE EIGHT TRACKS USING AS FOR TRACE OPTIMIZATION AND COMPARISON WITH THE BASE CONTROLLER

\begin{tabular}{|c|c|c|c|c|}
\hline Track & & Base & Path & Improv. \\
\hline 1 & Time & $29.383 \pm 0.146$ & $27.911 \pm 0.616$ & $5.01 \%$ \\
& \# Evals & & $544.86 \pm 130.10$ & \\
\hline 2 & Time & $29.840 \pm 0.103$ & $28.668 \pm 0.122$ & $3.92 \%$ \\
& \# Evals & & $577.2 \pm 124.88$ & \\
\hline 3 & Time & $19.499 \pm 0.842$ & $18.068 \pm 0.601$ & $7.34 \%$ \\
& \# Evals & & $451.23 \pm 78.66$ & \\
\hline 4 & Time & $23.688 \pm 0.251$ & $22.354 \pm 0.217$ & $5.63 \%$ \\
& \# Evals & & $542.36 \pm 138.19$ & \\
\hline 5 & Time & $28.765 \pm 0.869$ & $27.224 \pm 0.784$ & $5.35 \%$ \\
& \# Evals & & $953.56 \pm 141.68$ & \\
\hline 6 & Time & $50.544 \pm 0.777$ & $47.655 \pm 0.147$ & $5.71 \%$ \\
& \# Evals & & $599.46 \pm 130.85$ & \\
\hline 7 & Time & $43.106 \pm 0.164$ & $42.078 \pm 0.120$ & $2.38 \%$ \\
& \# Evals & & $739.66 \pm 103.62$ & \\
\hline 8 & Time & $20.360 \pm 0.998$ & $19.671 \pm 0.303$ & $3.38 \%$ \\
& \# Evals & & $1509.66 \pm 370.03$ & \\
\hline \multicolumn{4}{|c|}{ Average Improvement } & $4.84 \%$ \\
\hline
\end{tabular}

Table $\mathrm{V}$ shows the results (mean \pm deviation) for the time (in seconds) achieved by the base controller, the time related to the best trace found by the AS algorithm, the number of evaluations necessary to find the best trace, and the decrement in time achieved.

As can be seen, despite the base controller being implemented to achieve low times, the improvement is significant. In fact, the lap time is reduced on average by $4.84 \%$. However, the lack of precision in relation to speed estimation makes these times far from competitive, since an increment of $30 \%$ could be excesive on some curves, and would lead to the car taking them on the inside (and more slowly) or, on the other hand, a decrement of $30 \%$ for the inside part could lead to an excessive losts of time.

Regarding the number of evaluations, it can be observed that the search exceeds 1000 evaluations in only one case. This does not seem to be a huge computational effort.

However, when using the algorithm as a learning mechanism in real-time simulation environments, the number of evaluations necessary to find the best path is still too high.

Lastly, it is worth focusing on the results for the last track. As can be seen, despite being one of the "easy" tracks, the number of evaluations used to find the best trace is higher than it is for other tracks such as \# 6. Despite the fact that track \# 8 has less curves, some of them can be taken on either the inside or the outside with this speed setting without the car coming off. Because of this, these points are not discarded at early stages in the search and, therefore, the algorithm converges more slowly to a unique path.

\section{ADDING LOCAL SEARCH TO OPTIMIZE SPEED}

In Section $\mathrm{V}$ speed at each curve was calculated by means of the expression in Equation 1 and a heuristic modification that tried to reflect the effect of car position on such a speed. Despite the fact that lap time has been reduced, this estimation is very conservative and, therefore, substantial improvements can be made.

A simple local search procedure can be used to make a more precise adjustment. Given the base speed, calculated as mentioned above, the procedure consists of increasing it, for each point in a path, by successive intervals of $2.5 \%$ until the car crashes. Notice that, if $i_{c}$ is the number of steps necessary for estimating the maximum speed at point $p_{c}$, the total number of laps necessary to attain the minimum time for a path will be $L_{P}=\sum_{c=1}^{N} i_{c}$.

In order to test the effect of this local search procedure, it was applied over the traces obtained with the ACO algorithm described above. Table VI shows the results for the time in seconds and number of evaluations (mean \pm deviation) for the path algorithm without and with speed optimization, as well as the decrement in lap time.

TABLE II

RESULTS OBTAINED WHEN APPLYING SPEED OPTIMIZATION TO THE BEST TRACE FOUND BY THE AS ALGORITHM

\begin{tabular}{|c|c|c|c|c|}
\hline Track & & Path & Path \& Speed (I) & Improv. \\
\hline Track 1 & $\begin{array}{l}\text { Time } \\
\text { \# Evals }\end{array}$ & $\begin{array}{l}27.911 \pm 0.616 \\
544.86 \pm 130.10\end{array}$ & $\begin{array}{l}23.940 \pm 0.673 \\
566.23 \pm 130.94\end{array}$ & $14.22 \%$ \\
\hline Track 2 & $\begin{array}{c}\text { Time } \\
\text { \# Evals }\end{array}$ & $\begin{aligned} 28.668 & \pm 0.122 \\
577.2 & \pm 124.88\end{aligned}$ & $\begin{array}{l}24.603 \pm 0.122 \\
595.53 \pm 48.08\end{array}$ & $14.17 \%$ \\
\hline Track 3 & $\begin{array}{c}\text { Time } \\
\text { \# Evals }\end{array}$ & $\begin{array}{l}18.068 \pm 0.601 \\
451.23 \pm 78.66\end{array}$ & $\begin{array}{l}15.519 \pm 0.281 \\
466.53 \pm 78.28\end{array}$ & $14.10 \%$ \\
\hline Track 4 & $\begin{array}{c}\text { Time } \\
\text { \# Evals }\end{array}$ & $\begin{array}{l}22.354 \pm 0.217 \\
542.36 \pm 138.19\end{array}$ & $\begin{aligned} 19.235 & \pm 0.419 \\
559.4 & \pm 138.17\end{aligned}$ & $13.95 \%$ \\
\hline Track 5 & $\begin{array}{c}\text { Time } \\
\text { \# Evals }\end{array}$ & $\begin{array}{l}27.224 \pm 0.784 \\
953.56 \pm 141.68\end{array}$ & $\begin{aligned} \mathbf{2 3 . 3 0 3} & \pm 0.135 \\
980.4 & \pm 142.29\end{aligned}$ & $14.40 \%$ \\
\hline Track 6 & $\begin{array}{c}\text { Time } \\
\text { \# Evals }\end{array}$ & $\begin{array}{l}47.655 \pm 0.147 \\
599.46 \pm 130.85\end{array}$ & $\begin{array}{l}41.185 \pm 0.536 \\
625.43 \pm 130.53\end{array}$ & $13.57 \%$ \\
\hline Track 7 & $\begin{array}{c}\text { Time } \\
\text { \# Evals }\end{array}$ & $\begin{array}{l}42.078 \pm 0.120 \\
739.66 \pm 103.62\end{array}$ & $\begin{array}{l}36.081 \pm 0.573 \\
761.93 \pm 103.71\end{array}$ & $14.25 \%$ \\
\hline Track 8 & $\begin{array}{c}\text { Time } \\
\text { \# Evals }\end{array}$ & $\begin{aligned} 19.671 & \pm 0.303 \\
1509.66 & \pm 370.03\end{aligned}$ & $\begin{array}{l}16.862 \pm 0.556 \\
1530.9 \pm 370.53\end{array}$ & $14.27 \%$ \\
\hline & & & rage Improvement & $14.11 \%$ \\
\hline
\end{tabular}

As can be seen, the improvement achieved with speed optimization is quite significant. On average, lap time is reduced by $14.11 \%$.

If compared with the base controller, the lap time is reduced by $18.27 \%$.

Regarding the number of laps, it can be seen that, for each one of the tracks, the speed procedure adds around 20 more laps, which is not a significant amount even for the real-time case.

As mentioned in Section III the trace and speed are quite interdependent. In fact, the goodness of a trace depends on the maximum speed that can be reached with this path.

With the said algorithm, traces were evaluated without taking into account the maximum speed. Then, once the best trace was found, speed was optimized. However, there is a possibility that paths which lead to worst times with the basic speed setting, lead to good times when using the speed optimization procedure. Therefore, it seems reasonable to evaluate each one of the paths by applying the speed optimization procedure to it.

According to the local search procedure described above, speed optimization requires $L_{P}=\sum_{c=1}^{N} i_{c}$ laps. This fact could make the evaluation of a path very costly, but some effort can be saved in the application of this procedure.

As the maximum speed at a certain point $p_{c}$ depends on the angle of the curve $c$, it also depends on both $p_{c-1}$ and $p_{c+1}$. However, it does not depend on the rest of the points. 
Therefore, for each triplet $\left\langle p_{c-1}, p_{c}, p_{c+1}\right\rangle$, the maximum speed need only be estimated once.

Taking this into account, in the evaluation process of a certain path $P$ only the speed for triplets not yet optimized needs to be optimized. This fact helps to reduce the number of evaluations substantially. Moreover, when using the AS algorithm only those paths built by ants must be optimized. As these paths tend to follow the same stretches and converge, optimization only needs to be performed at first stages of the search, since afterwards, speed will already have been calculated for each triplet.

Figure 7 shows the procedure for the speed adjustment which is used with the AS algorithm for trace optimization.

\section{Speed optimization}

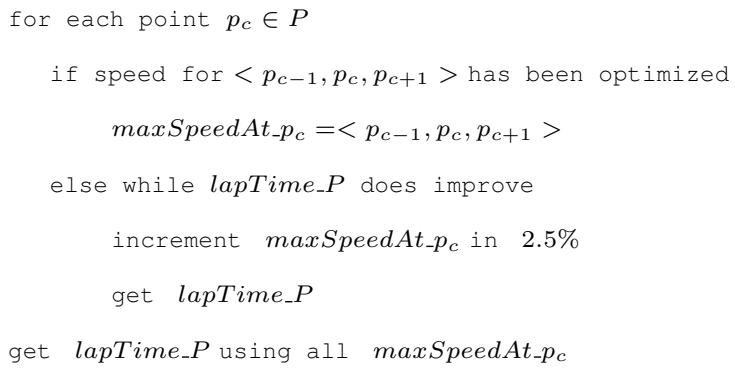

Fig. 7. Speed optimization procedure

Table VI shows the results, mean \pm deviation of the best time and mean \pm deviation of the number of evaluations, for the algorithm which applies speed optimization to the best trace found (I), and the one which applies this optimization to each trace (II). The improvement in the time achieved with the second algorithm is also shown.

As can be seen, the improvement, in terms of lap time, is quite small, as it is only reduced by $0.35 \%$ on average. The number of evaluations, however, increases significantly.

TABLE III

RESULTS OBTAINED WHEN APPLYING SPEED OPTIMIZATION TO ALL TRACES EVALUATED

\begin{tabular}{|c|c|c|c|c|}
\hline Track & & Trace \& Speed (I) & Trace \& Speed (II) & Improvement \\
\hline Track 1 & $\begin{array}{l}\text { Time } \\
\text { \# Evals }\end{array}$ & $\begin{array}{l}23.940 \pm 0.673 \\
566.23 \pm 130.94\end{array}$ & $\begin{array}{l}\mathbf{2 3 . 8 3 6} \pm 0.140 \\
1003.4 \pm 63.48\end{array}$ & $0.43 \%$ \\
\hline Track 2 & $\begin{array}{c}\text { Time } \\
\text { \# Evals }\end{array}$ & $\begin{array}{l}24.603 \pm 0.122 \\
595.53 \pm 48.08\end{array}$ & $\begin{array}{l}\mathbf{2 4 . 5 4 8} \pm 0.723 \\
970.46 \pm 57.67\end{array}$ & $0.22 \%$ \\
\hline Track 3 & $\begin{array}{c}\text { Time } \\
\text { \# Evals }\end{array}$ & $\begin{array}{l}15.519 \pm 0.281 \\
466.53 \pm 78.28\end{array}$ & $\begin{aligned} \mathbf{1 5 . 4 9 4} & \pm 0.598 \\
763.8 & \pm 55.59\end{aligned}$ & $0.26 \%$ \\
\hline Track 4 & $\begin{array}{c}\text { Time } \\
\text { \# Evals } \\
\end{array}$ & $\begin{aligned} 19.235 & \pm 0.419 \\
559.4 & \pm 138.17\end{aligned}$ & $\begin{array}{l}\mathbf{1 9 . 1 9 2} \pm 0.659 \\
1605.3 \pm 230.37\end{array}$ & $0.22 \%$ \\
\hline Track 5 & $\begin{array}{c}\text { Time } \\
\text { \# Evals }\end{array}$ & $\begin{aligned} \mathbf{2 3 . 3 0 3} & \pm 0.135 \\
980.4 & \pm 142.29\end{aligned}$ & $\begin{array}{r}23.331 \pm 0.143 \\
954.7 \pm 54.22\end{array}$ & $-0.12 \%$ \\
\hline Track 6 & $\begin{array}{c}\text { Time } \\
\text { \# Evals }\end{array}$ & $\begin{array}{l}41.185 \pm 0.536 \\
625.43 \pm 130.53\end{array}$ & $\begin{array}{r}\mathbf{4 0 . 9 5 8} \pm 0.289 \\
1112.76 \pm 65.57\end{array}$ & $0.55 \%$ \\
\hline Track 7 & $\begin{array}{c}\text { Time } \\
\text { \# Evals }\end{array}$ & $\begin{array}{l}36.081 \pm 0.573 \\
761.93 \pm 103.71\end{array}$ & $\begin{array}{l}\mathbf{3 6 . 0 3 2} \pm 0.127 \\
1028.5 \pm 64.01\end{array}$ & $0.13 \%$ \\
\hline Track 8 & $\begin{array}{c}\text { Time } \\
\text { \# Evals }\end{array}$ & $\begin{array}{l}16.862 \pm 0.556 \\
1530.9 \pm 370.53\end{array}$ & $\begin{array}{r}\mathbf{1 6 . 6 4 8} \pm 0.152 \\
1065.66 \pm 75.63 \\
\end{array}$ & $1.26 \%$ \\
\hline & & & verage Improvement & $0.35 \%$ \\
\hline
\end{tabular}

Therefore, this algorithm does not seem suitable as a learning technique for a controller in a real-time environment.

The small difference between this algorithm (II) and the former one (I) may be due to several factors.

First of all, it seems that the first algorithm does not discard, during the search, traces which would be substantially improved by the speed optimization procedure. At least, the paths are quite similar. That leads us to think that the initial speed estimation is suitable to find the right traces.

On the other hand, once a good trace has been found and the speed has been optimized, the possible improvements are quite small. Since the car always takes curves the same way (when it is placed at the entry point $p_{c}$, it moves towards the middle point of the exit), the time reduction due to the trace is, in some sense, limited. Improving the way curves are taken by, for instance, defining more points for the trajectory on them or estimating the steer angle, could make these small differences more significant.

\section{CONCLUSIONS AND FUTURE WORK}

This article has presented a method for improving some features of a car racing controller so that it reduces the lap time on a known circuit. In particular, we have focused on finding the right trace around the track and optimizing the speed on each curve.

In order to determine the traces an ACO algorithm, the Ant System, was used. This kind of algorithms are suitable for solving similar problems when heuristic information is available, as is the case here.

Despite the fact that results obtained with a heuristic setting of the speed improve on the original ones, results were still not very competitive. Therefore, a local search procedure was been used to improve the speed estimation. This was done in two ways:

In the first one, the procedure was applied to the trace found by the AS algorithm. The improvement in this case was quite significant. Moreover, the number of additional evaluations required by the procedure is reduced. This is due to the fact that the problem of speed maximization is decomposable.

With the second algorithm, even if there is a large increment in the number of evaluations, results do not improve significatively.

In conclusion, it could be said that some improvement can be achieved when the car moves along a good trace, even with the heuristic setting of speed. However, the use of the speed optimization procedure reduces the lap time without needing a big number of additional evaluations.

The proposed techniques could also be considered as learning mechanisms. Thus, instead of viewing them as an external optimization algorithm, their nature allows them to be implemented as an internal part of the controller so that it reduces the lap time without external information. However, the number of evaluations is still too high when considering real-time simulation environments.

As future work, intend to focus on this issue. One of the ways of dealing with this problem consists of studying other 
parameter configurations for the ACO algorithm or even using other approaches as greeedy algorithms. Moreover, some other techniques that use previous experience could be used as a way of reducing the search space and learning traces and speeds more quickly.

Besides future work related with convergence, there are some other factors that should be studied to obtain a competitive controller:

- Controlling the braking distance so that maximum power is mantained as long as possible before setting the speed for a curve.

- Improving the way curves are taken: different points around the curve or even the steering radius can be learnt in order to gain some speed.

\section{ACKNOWLEDGMENTS}

This study has been partially aided by the Consejería de Educación y Ciencia (JCCM) under project PCI08-00488577.

\section{REFERENCES}

[1] "IEEE Symposium on Computation Intelligence and Games 2007." http://csapps.essex.ac.uk/cig/2007/, 2007.

[2] "IEEE World Congress on Computational Intelligence 2008." http://www.wcci2008.org/, 2008.
[3] J. Togelius and S. M. Lucas., "Evolving controllers for simulated car racing," in Proceedings of IEEE Congress on Evolutionary Computation, 2005.

[4] J. Togelius and S. M. Lucas, "Evolving robust and specialized car racing skills," in Proceedings of the IEEE Congress on Evolutionary Computation, 2006. IEEE Press, 2006.

[5] A. Agapitos, J. Togelius, and S. M. Lucas, "Evolving controllers for simulated car racing using object oriented genetic programming," in GECCO '07: Proceedings of the 9th annual conference on Genetic and evolutionary computation. New York, NY, USA: ACM, 2007, pp. $1543-1550$

[6] E. Bonabeau, M. Dorigo, and G. Theraulaz, Swarm Intelligence: From natural to artificial systems. Oxford University Press, 1999.

[7] M. Dorigo, V. Maniezzo, and A. Colorni, "The Ant System: Optimization by a colony of cooperating agents," IEEE Transactions on Systems, Man, and Cybernetics Part B. Cybernetics, vol. 26, no. 1, pp. 29-41, 1996. [Online]. Available: citeseer.ist.psu.edu/dorigo96ant.html

[8] "TORCS: The Open Racing Car Simulator," http://torcs. sourceforge.net/.

[9] J. Togelius, "Optimization, imitation and innovation: Computational intelligence and games," Ph.D. dissertation, Departament of Computing and Electronic Systems, University of Essex, 2007.

[10] J. L. Deneubourg, S. Aron, S. Goss, and J. M. Pasteels, "The self organizing exploratory pattern of the argentine ant," Journal of Insect Behavior, vol. 3, no. 2, p. 159 168, 1990.

[11] M. Dorigo and L. M. Gambardella, "Ant colony system: A cooperative learning approach to the traveling salesman problem," IEEE Transactions on Evolutionary Computation, vol. 1, no. 1, pp. 53-66, April 1997. [Online]. Available: citeseer.ist.psu.edu/article/dorigo96ant.html

[12] T. Stützle and H. H. Hoos, "Max-min ant system," Future Generation Comp. Syst., vol. 16, no. 8, pp. 889-914, 2000. 